\title{
Cytomorphological Study in Genus Cenchrus L.: An Important Medicinal Plant from North India (Family: Poaceae)
}

\author{
Akshita Dhaliwal $^{1}$, Rajdeep Singh Dhaliwal ${ }^{1}$, \\ Navjot Kaur ${ }^{2 *}$ and Raghbir Chand Gupta ${ }^{2}$ \\ ${ }^{1}$ Post Graduate Department of Agriculture, G.S.S.D.G.S. Khalsa College, Patiala 147001, India \\ ${ }^{2}$ Department of Botany, Punjabi University, Patiala 147002, India
}

Received September 23, 2017; accepted October 8, 2017

\begin{abstract}
Summary The present study deals with the four species of genus Cenchrus L. based on population analysis from different localities in Haryana and adjoining hills of Shiwalik area. These species i.e., C. biflorus, C. ciliaris, C. pennisetiformis and C. setigerus differ from each other morphologically as well as cytologically. In $C$. biflorus, cytological studies have been made on 23 populations from the study area and three cytotypes $(n=16$, 17 and 18) are detected. In C. ciliaris, most commonly found species of the genus, 48 populations were studied and four cytotypes with chromosome numbers $n=16,17,18$ and 36 are found. The population analysis revealed that the tetraploid cytotype $(n=18)$ based on $x=9$ is better adapted as compared to the other three cytotypes. The tetraploid and aneuploid cytotypes are almost morphologically similar, whereas octaploid shows lot of gigantism. All the studied cytotypes of $C$. ciliaris show meiotic abnormalities. In $C$. pennisetiformis, two cytotypes with $n=17$ and 18 are observed, which were found to be morphologically similar with abnormal meiotic course. In this species, $2 n=34$ is a new chromosome report. The fourth species $C$. setigerus, also shows similar cytotypes with $n=17$ and 18 , based on the study of 49 populations. These cytotypes are morphologically indistinct and show overlapping morphometric characters.
\end{abstract}

Key words Cenchrus species, Meiotic abnormality, Cytotype, Aneuploid, Haryana and Shiwalik Hills.

Family Poaceae is the third largest family among the angiosperms with about 898 genera and 10000 species (Tzvelev 1989), which is represented in India by about 268 genera and 1200 species (Moulik 1997). The family has vast economic importance and it also exhibits lot of cytological variation. The family exhibits lot of cytological diversity with most documented cytological evidences such as polyploidy, aneuploidy, apomixis, etc.

The genus Cenchrus belongs to the tribe Paniceae of Poaceae. The genus is represented with 25 species (Watson and Dallwitz 1992) and seven species are reported from Indian subcontinent (Bor 1960). It is distributed throughout the tropical and subtropical parts in world. The main characteristic features of the genus are: presence of involucres of spines and rigid bristles united at the base into a hard cup. Taxonomically, the genus Cenchrus is separated from the genus Pennisetum by involucral bristles fused at their point of origin (Nath et al. 1970). Some of the species of the genus are used as fodder and three species (C. ciliaris, C. echinatus, C. myosuroides) are considered as important weeds as highly nutritious and palatable fodder (Clayton and Renvoize 1986). Due to its resilience to harsh environmental con-

\footnotetext{
* Corresponding author, e-mail: navjot21188@gmail.com DOI: $10.1508 /$ cytologia. 83.45
}

ditions, it is cultivated as one of the major forage grasses in India (Bhat et al. 2001).

The research on the cytology of grasses had been undertaken from Haryana and Shiwalik hills, Rajasthan, Kangra district, Pangi valley of Kullu, Kinnaur district and Kashmir (see Kaur and Gupta 2016a, 2016b, Gupta et al. 2017).

\section{Materials and methods}

Materials for the meiotic studies are collected from number of localities from Haryana and adjoining Shiwalik hills (Table 1). Voucher specimens are deposited in the Herbarium, Department of Botany, Punjabi University, Patiala (PUN). Meiotic studies are carried out through standard smearing technique from young panicles fixed in Carnoy's fixative. For the analysis of meiotic abnormalities, a large number of PMCs was counted for each type of anomaly. Pollen fertility is estimated through glycero-acetocarmine stainability. Wellfilled pollen grains with stained nuclei were taken as apparently viable/fertile while shriveled and unstained pollen grains are counted as sterile. Pollen grain size is measured using occulomicrometre. Photomicrographs of chromosome counts are taken from freshly prepared slides using Nikon 80i eclipse digital imaging system. 
Table 1. Data regarding the taxa with population, locality with altitude, accession number, meiotic chromosome number, pollen fertility, pollen size and ploidy level of the studied species of Cenchrus.

\begin{tabular}{|c|c|c|c|c|c|c|c|}
\hline Taxa & Locality with altitude & $\begin{array}{c}\text { Accession } \\
\text { number } \\
\text { (PUN) }\end{array}$ & $\begin{array}{c}\text { Chromosome } \\
\text { number }(n)\end{array}$ & $\begin{array}{c}\text { Pollen } \\
\text { fertility } \\
(\%)\end{array}$ & $\begin{array}{l}\text { Pollen size } \\
\qquad(\mu \mathrm{m})\end{array}$ & Ploidy level & $\begin{array}{l}\text { Remarks } \\
\text { in meiosis }\end{array}$ \\
\hline \multicolumn{8}{|c|}{ Cenchrus biflorus Roxb. } \\
\hline \multicolumn{8}{|c|}{ Cytotype A $(n=16)$} \\
\hline $\mathrm{P}-1$ & Nanhari, Yamunanagar (255 m) & 49920 & 16 & 25.7 & $24.3-26.3 \times 24.4-26.3$ & $4 x$ & $\mathrm{ABN}$ \\
\hline P-2 & Kalka, Shiwaliks (624 m) & 49927 & 16 & 37.2 & $18.8-20.6 \times 20.6$ & $4 x$ & $\mathrm{ABN}$ \\
\hline \multicolumn{8}{|c|}{ Cytotype B $(n=17)$} \\
\hline $\mathrm{P}-3$ & Guhla, Kaithal (219 m) & 49919 & 17 & 15.4 & $24.3-26.3 \times 22.5-24.4$ & $4 x$ (Aneu.) & $\mathrm{ABN}$ \\
\hline P-4 & Naror, Kaithal (219 m) & 49931 & 17 & 49.6 & $20.6-22.5 \times 22.5$ & $4 x$ (Aneu.) & $\mathrm{ABN}$ \\
\hline P-5 & Cheeka, Kaithal (219m) & 49932 & 17 & 52.4 & $24.4-26.3 \times 26.3$ & $4 x$ (Aneu.) & $\mathrm{ABN}$ \\
\hline P-6 & Pehowa Road, Kaithal (219m) & 49933 & 17 & 64.6 & $24.4-26.3 \times 24.4-26.3$ & $4 x$ (Aneu.) & $\mathrm{ABN}$ \\
\hline \multicolumn{8}{|c|}{ Cytotype $\mathrm{C}(n=18)$} \\
\hline P-7 & CCS Univ., Hissar (216 m) & 49921 & 18 & 12.5 & $24.4-26.3 \times 26.3$ & $4 x$ & $\mathrm{ABN}$ \\
\hline P-8 & Lalru, Ambala (272 m) & 49922 & 18 & 34.6 & $20.6-22.5 \times 20.6-22.5$ & $4 x$ & $\mathrm{ABN}$ \\
\hline P-9 & Nizampur, Mahendragarh (261 m) & 49923 & 18 & 18.4 & $18.8-22.5 \times 18.8$ & $4 x$ & $\mathrm{ABN}$ \\
\hline P-10 & Nahan, Sirmour (932 m) & 49924 & 18 & 52.7 & $20.6-22.5 \times 22.5$ & $4 x$ & $\mathrm{ABN}$ \\
\hline P-11 & Thanesar, Kurukshetra (350 m) & 49925 & 18 & 34.7 & $24.4-26.3 \times 24.4-26.3$ & $4 x$ & $\mathrm{ABN}$ \\
\hline P-17 & Narayangarh, Ambala (272 m) & 49935 & 18 & 25.4 & $20.6-22.5 \times 20.6-22.5$ & $4 x$ & $\mathrm{ABN}$ \\
\hline P-18 & Firozpur, Jhirka, Mewat (200m) & 49936 & 18 & 42.8 & $30.0-33.8 \times 30.0-33.8$ & $4 x$ & $\mathrm{ABN}$ \\
\hline P-19 & Agroha, Hissar $(216 \mathrm{~m})$ & 49937 & 18 & 39.2 & $30.3-33.8 \times 33.0$ & $4 x$ & $\mathrm{ABN}$ \\
\hline P-20 & Nilokheri, Karnal (252 m) & 49938 & 18 & 50.2 & $26.3-30.0 \times 30.0$ & $4 x$ & $\mathrm{~N}$ \\
\hline $\mathrm{P}-21$ & HAU, Hissar $(216 \mathrm{~m})$ & 49939 & 18 & 62.4 & $22.5-24.4 \times 24.4$ & $4 x$ & $\mathrm{~N}$ \\
\hline P-22 & Radaur, Yamunanagar (255 m) & 49940 & 18 & 48.2 & $26.3 \times 24.4-26.3$ & $4 x$ & $\mathrm{ABN}$ \\
\hline P-23 & Ganaur, Sonipat $(219 \mathrm{~m})$ & 49941 & 18 & 52.7 & $22.5-24.4 \times 22.5-24.4$ & $4 x$ & $\mathrm{~N}$ \\
\hline \multicolumn{8}{|c|}{ C. ciliaris $\mathrm{L}$. } \\
\hline \multicolumn{8}{|c|}{ Cytotype A $(n=16)$} \\
\hline $\mathrm{P}-1$ & Indri, Karnal (252 m) & 49874 & 16 & 0.0 & $24.4-26.3 \times 22.5-24.4$ & $4 x$ & $\mathrm{ABN}$ \\
\hline P-2 & Babain, Kurukshetra (350 m) & 49912 & 16 & 50.4 & $22.5-24.4 \times 20.6-22.5$ & $4 x$ & $\mathrm{~N}$ \\
\hline \multicolumn{8}{|c|}{ Cytotype B $(n=17)$} \\
\hline P-3 & HAU, Hissar $(216 \mathrm{~m})$ & 49881 & 17 & 54.7 & $26.3-30.0 \times 30.0$ & $4 x$ (Aneu.) & $\mathrm{ABN}$ \\
\hline \multicolumn{8}{|c|}{ Cytotype C $(n=18)$} \\
\hline P-4 & Mullana, Ambala (272 m) & 49871 & 18 & 0.0 & $20.6-22.5 \times 20.6-22.5$ & $4 x$ & $\mathrm{ABN}$ \\
\hline $\mathrm{P}-5$ & Barog, Solan (1350m) & 49872 & 18 & 39.3 & $22.5-39.4 \times 22.5-39.4$ & $4 x$ & $\mathrm{ABN}$ \\
\hline P-6 & Nanhari, Yamunanagar (255 m) & 49873 & 18 & 23.7 & $22.5-24.4 \times 22.5$ & $4 x$ & $\mathrm{ABN}$ \\
\hline P-7 & Guhla, Kaithal (219m) & 49875 & 18 & 0.0 & $22.5-24.4 \times 20.6-22.5$ & $4 x$ & $\mathrm{ABN}$ \\
\hline P-8 & CCS, Univ, Hissar (216m) & 49876 & 18 & 3.75 & $22.5-24.4 \times 18.8-22.5$ & $4 x$ & $\mathrm{ABN}$ \\
\hline P-9 & Mirpur, Rewari (242 m) & 49877 & 18 & 27.9 & $18.8-22.5 \times 18.8$ & $4 x$ & $\mathrm{ABN}$ \\
\hline P-10 & KUK, Kurukshetra (350 m) & 49878 & 18 & 0.0 & $24.4-26.3 \times 22.5-26.3$ & $4 x$ & $\mathrm{ABN}$ \\
\hline P-11 & Jyotisar, Kurukshetra (350 m) & 49879 & 18 & 6.9 & $26.3-33.8 \times 26.3-30.0$ & $4 x$ & $\mathrm{ABN}$ \\
\hline P-12 & Agroha, Hissar (216m) & 49880 & 18 & 22.8 & $22.5-24.4 \times 22.5-24.4$ & $4 x$ & $\mathrm{ABN}$ \\
\hline P-13 & Gharaunda, Karnal (252 m) & 49882 & 18 & 55.2 & $22.5-24.4 \times 20.6-22.5$ & $4 x$ & $\mathrm{~N}$ \\
\hline P-14 & Narnaul, Mahendragarh (261 m) & 49883 & 18 & 35.9 & $22.5-24.4 \times 22.5$ & $4 x$ & $\mathrm{ABN}$ \\
\hline P-15 & Sanauli, Panipat $(218 \mathrm{~m})$ & 49884 & 18 & 65.4 & $20.6-22.5 \times 20.6-22.5$ & $4 x$ & $\mathrm{~N}$ \\
\hline P-16 & Devsar, Bhiwani (224 m) & 49885 & 18 & 37.4 & $24.4-26.3 \times 22.5-26.3$ & $4 x$ & $\mathrm{ABN}$ \\
\hline P-17 & Radaur, Yamunanagar (255 m) & 49886 & 18 & 30.5 & $18.8-22.5 \times 18.8$ & $4 x$ & $\mathrm{ABN}$ \\
\hline P-18 & Lalru, Ambala (272m) & 49887 & 18 & 52.9 & $26.3-30.0 \times 30.0$ & $4 x$ & $\mathrm{ABN}$ \\
\hline P-19 & Naggal, Ambala (272 m) & 49888 & 18 & 42.4 & $22.5-24.4 \times 22.5$ & $4 x$ & $\mathrm{ABN}$ \\
\hline P-20 & Samalkha, Panipat (218 m) & 49889 & 18 & 67.4 & $26.3-33.8 \times 26.3-30.0$ & $4 x$ & $\mathrm{~N}$ \\
\hline $\mathrm{P}-21$ & Gohana, Sonipat (219m) & 49890 & 18 & 65.2 & $26.3-30.0 \times 26.3-30.0$ & $4 x$ & $\mathrm{ABN}$ \\
\hline P-22 & Sunariya, Rohtak (213 m) & 49891 & 18 & 58.4 & $24.4-26.3 \times 26.3$ & $4 x$ & $\mathrm{ABN}$ \\
\hline P-23 & Bahadurgarh, Jhajjar (219m) & 49892 & 18 & 62.3 & $18.8-20.6 \times 18.8-20.6$ & $4 x$ & $\mathrm{ABN}$ \\
\hline P-24 & Nizampur, Mahendragarh (261 m) & 49893 & 18 & 49.7 & $26.3-30.0 \times 30.0$ & $4 x$ & $\mathrm{ABN}$ \\
\hline P-25 & Shazadpur, Ambala (272 m) & 49894 & 18 & 72.6 & $22.5-24.4 \times 22.5$ & $4 x$ & $\mathrm{~N}$ \\
\hline P-26 & Parwanoo, Solan $(1350 \mathrm{~m})$ & 49895 & 18 & 60.7 & $20.6-22.5 \times 20.6-22.5$ & $4 x$ & $\mathrm{ABN}$ \\
\hline P-27 & Trilokpur, Shiwaliks (932 m) & 49896 & 18 & 74.0 & $26.3-33.8 \times 26.3-30.0$ & $4 x$ & $\mathrm{~N}$ \\
\hline P-28 & Kala amb, Ambala (272 m) & 49897 & 18 & 59.2 & $18.8-20.6 \times 18.8-20.6$ & $4 x$ & $\mathrm{~N}$ \\
\hline P-29 & Mandi Dabwali, Sirsa (204 m) & 49898 & 18 & 31.4 & $22.5-24.4 \times 22.5-24.4$ & $4 x$ & $\mathrm{~N}$ \\
\hline P-30 & Gohana, Sonipat $(219 \mathrm{~m})$ & 49899 & 18 & 26.7 & $24.4-26.3 \times 24.4-26.3$ & $4 x$ & $\mathrm{ABN}$ \\
\hline P-31 & Naror, Kaithal (219m) & 49900 & 18 & 0.0 & $24.4-26.3 \times 24.4$ & $4 x$ & $\mathrm{ABN}$ \\
\hline P-32 & Shahpur, Ambala (272 m) & 49901 & 18 & 52.6 & $18.8-22.5 \times 18.8$ & $4 x$ & $\mathrm{ABN}$ \\
\hline P-33 & Nilokheri, Karnal (252 m) & 49902 & 18 & 32.9 & $30.0 \times 30.0$ & $4 x$ & $\mathrm{~N}$ \\
\hline P-34 & Narwana, Jind (226 m) & 49903 & 18 & 0.0 & $26.3-30.0 \times 30.0$ & $4 x$ & $\mathrm{ABN}$ \\
\hline P-35 & Jagadhri, Yamunanagar (255 m) & 49904 & 18 & 26.4 & $26.3-33.8 \times 26.3-30.0$ & $4 x$ & $\mathrm{ABN}$ \\
\hline P-36 & Kalanaur, Yamunanagar $(255 \mathrm{~m})$ & 49905 & 18 & 25.0 & $26.3-30 . \times 26.3-30.0$ & $4 x$ & $\mathrm{~N}$ \\
\hline P-37 & Kalka, Shiwaliks (624 m) & 49906 & 18 & 17.6 & $24.4-26.3 \times 22.5-26.3$ & $4 x$ & $\mathrm{ABN}$ \\
\hline P-38 & Barara, Ambala (272 m) & 49907 & 18 & 27.6 & $24.4-26.3 \times 22.5-24.4$ & $4 x$ & $\mathrm{ABN}$ \\
\hline P-39 & Firozpur Jhirka, Mewat (200 m) & 49908 & 18 & 37.5 & $22.5-24.4 \times 18.8-22.5$ & $4 x$ & $\mathrm{ABN}$ \\
\hline P-40 & Thanesar, Kurukshetra (350 m) & 49909 & 18 & 15.7 & $24.4-26.3 \times 22.5$ & $4 x$ & $\mathrm{ABN}$ \\
\hline P-41 & Ballabgarh, Faridabad (197 m) & 49910 & 18 & 24.6 & $22.5-24.4 \times 22.5-24.4$ & $4 x$ & $\mathrm{ABN}$ \\
\hline
\end{tabular}


Table 1. Continued

\begin{tabular}{|c|c|c|c|c|c|c|c|}
\hline Taxa & Locality with altitude & $\begin{array}{l}\text { Accession } \\
\text { number } \\
(\mathrm{PUN})\end{array}$ & $\begin{array}{l}\text { Chromosome } \\
\text { number }(n)\end{array}$ & $\begin{array}{l}\text { Pollen } \\
\text { fertility } \\
(\%)\end{array}$ & $\begin{array}{l}\text { Pollen size } \\
\qquad(\mu \mathrm{m})\end{array}$ & Ploidy level & $\begin{array}{l}\text { Remarks } \\
\text { in meiosis }\end{array}$ \\
\hline P-42 & Ratia, Fatehbad (207 m) & 49911 & 18 & 40.6 & $22.5-24.4 \times 22.5$ & $4 x$ & $\mathrm{ABN}$ \\
\hline P-43 & Narayangarh, Ambala (272 m) & 49913 & 18 & 67.2 & $26.3-33.8 \times 26.3-30.0$ & $4 x$ & $\mathrm{~N}$ \\
\hline P-44 & Cheeka, Kaithal (219m) & 49914 & 18 & 42.3 & $26.3-30.0 \times 26.3-30.0$ & $4 x$ & $\mathrm{~N}$ \\
\hline P-45 & Nahan, Sirmour (932 m) & 49915 & 18 & 65.4 & $20.6-22.5 \times 20.6-22.5$ & $4 x$ & $\mathrm{~N}$ \\
\hline P-46 & Panjab Univ, Chandigarh (308 m) & 49916 & 18 & 45.6 & $22.5-22.4 \times 22.5$ & $4 x$ & $\mathrm{ABN}$ \\
\hline P-47 & Pinjour, Panchkula (365 m) & 49917 & 18 & 52.6 & $24.4-26.3 \times 22.5-24.4$ & $4 x$ & $\mathrm{ABN}$ \\
\hline \multicolumn{8}{|c|}{ Cytotype $\mathrm{D}(n=36)$} \\
\hline $\mathrm{P}-48$ & Jabli, Shivaliks (900 m) & 49918 & 36 & 82.7 & $37.5-39.4 \times 37.5-39.4$ & $8 x$ & $\mathrm{~N}$ \\
\hline Cytotype A $(n=17)$ & \multicolumn{7}{|c|}{ C. pennisetiformis Hochst. et. Steud. } \\
\hline P-1 & Kalka, Shiwaliks (624 m) & 49991 & 17 & 19.9 & $22.5-24.4 \times 22.5$ & $4 x$ (Aneu.) & $\begin{array}{l}\text { First chromosome } \\
\text { report } \mathrm{ABN}\end{array}$ \\
\hline \multicolumn{8}{|c|}{ Cytotype B $(n=18)$} \\
\hline $\mathrm{P}-2$ & Sanawar, Solan (1350 m) & 49990 & 18 & 27.8 & $18.8-22.5 \times 18.8$ & $4 x$ & $\mathrm{ABN}$ \\
\hline \multicolumn{8}{|c|}{ C. setigerus Vahl. } \\
\hline \multicolumn{8}{|c|}{ Cytotype A $(n=17)$} \\
\hline P-1 & Kalanaur, Yamunanagar (255 m) & 49976 & 17 & 17.5 & $22.5-24.4 \times 22.5-24.4$ & $4 x$ (Aneu.) & $\mathrm{ABN}$ \\
\hline $\mathrm{P}-2$ & Kalka, Shiwaliks (624 m) & 49977 & 17 & 0.0 & $22.5-24.4 \times 22.5-24.4$ & $4 x$ (Aneu.) & $\mathrm{ABN}$ \\
\hline $\mathrm{P}-3$ & Barara, Ambala (272 m) & 49978 & 17 & 15.6 & $22.5-24.4 \times 22.5-24.4$ & $4 x$ (Aneu.) & $\mathrm{ABN}$ \\
\hline P-4 & Firozpur Jhirka, Mewat (200 m) & 49979 & 17 & 22.7 & $22.5-24.4 \times 24.4$ & $4 x$ (Aneu.) & $\mathrm{ABN}$ \\
\hline P-5 & Thanesar, Kurukshetra (350 m) & 49980 & 17 & 30.7 & $24.4-26.3 \times 24.4-26.3$ & $4 x$ (Aneu.) & $\mathrm{ABN}$ \\
\hline P-6 & Ballabgarh, Faridabad (197 m) & 49981 & 17 & 24.9 & $24.4-26.3 \times 24.4-26.3$ & $4 x$ (Aneu.) & $\mathrm{ABN}$ \\
\hline P-7 & Ratia, Fatehbad $(207 \mathrm{~m})$ & 49982 & 17 & 12.6 & $22.5-24.4 \times 22.5-24.4$ & $4 x$ (Aneu.) & $\mathrm{ABN}$ \\
\hline P-8 & Babaur, Kurukshetra (350 m) & 49983 & 17 & 21.9 & $22.5-24.4 \times 22.5-24.4$ & $4 x$ (Aneu.) & $\mathrm{ABN}$ \\
\hline P-9 & Narayangarh, Ambala (272 m) & 49984 & 17 & 30.6 & $24.4-26.3 \times 24.4$ & $4 x$ (Aneu.) & $\mathrm{ABN}$ \\
\hline P-10 & Cheeka, Kaithal (219m) & 49985 & 17 & 16.4 & $24.4-26.3 \times 24.4-26.3$ & $4 x$ (Aneu.) & $\mathrm{ABN}$ \\
\hline P-11 & Nahan, Sirmour (932 m) & 49986 & 17 & 0.0 & $24.4-26.3 \times 22.5-24.4$ & $4 x$ (Aneu.) & $\mathrm{ABN}$ \\
\hline P-12 & Panjab Univ., Chandigarh (308 m) & 49987 & 17 & 24.8 & $22.5-24.4 \times 22.5-24.4$ & $4 x$ (Aneu.) & $\mathrm{ABN}$ \\
\hline P-13 & Pinjour, Panchkula (365 m) & 49988 & 17 & 13.2 & $24.4-26.3 \times 24.4-26.3$ & $4 x$ (Aneu.) & $\mathrm{ABN}$ \\
\hline P-14 & Jabli, Shiwaliks (900 m) & 49989 & 17 & 0.0 & $24.4-26.3 \times 24.4-26.3$ & $4 x$ (Aneu.) & $\mathrm{ABN}$ \\
\hline P-15 & Ganaur, Sonipat (219 m) & 49992 & 17 & 26.1 & $22.5-24.4 \times 22.5-24.4$ & $4 x$ (Aneu.) & $\mathrm{ABN}$ \\
\hline \multicolumn{8}{|c|}{ Cytotype B $(n=18)$} \\
\hline P-16 & Mullana, Ambala (272 m) & 49942 & 18 & 0.0 & $22.5-28.1 \times 22.5-26.3$ & $4 x$ & $\mathrm{~N}$ \\
\hline P-17 & Barog, Solan $(1350 \mathrm{~m})$ & 49943 & 18 & 12.5 & $22.5-24.4 \times 24.4$ & $4 x$ & $\mathrm{~N}$ \\
\hline P-18 & Nanhari, Yamunanagar (255 m) & 49944 & 18 & 68.9 & $24.4-26.3 \times 22.5-24.4$ & $4 x$ & $\mathrm{ABN}$ \\
\hline P-19 & Indri, Karnal (252 m) & 49945 & 18 & 52.7 & $22.5-24.4 \times 22.5-24.4$ & $4 x$ & $\mathrm{ABN}$ \\
\hline P-20 & Guhla, Kaithal (219m) & 49946 & 18 & 47.4 & $24.4-26.3 \times 24.4-26.3$ & $4 x$ & $\mathrm{~N}$ \\
\hline P-21 & CCS Univ., Hissar (216 m) & 49947 & 18 & 17.5 & $24.4-28.1 \times 22.5-24.4$ & $4 x$ & $\mathrm{ABN}$ \\
\hline P-22 & Mirpur, Rewari (242 m) & 49948 & 18 & 25.4 & $24.4-26.3 \times 24.4-26.3$ & $4 x$ & $\mathrm{ABN}$ \\
\hline P-23 & KUK, Kurukshetra (350 m) & 49949 & 18 & 24.4 & $22.5-24.4 \times 22.5-24.4$ & $4 x$ & $\mathrm{ABN}$ \\
\hline P-24 & Jyotisar, Kurukshetra (350 m) & 49950 & 18 & 15.9 & $24.4-26.3 \times 24.4-26.3$ & $4 x$ & $\mathrm{ABN}$ \\
\hline P-25 & Agroha, Hissar $(216 \mathrm{~m})$ & 49951 & 18 & 32.6 & $22.5-24.4 \times 22.5-24.4$ & $4 x$ & $\mathrm{ABN}$ \\
\hline P-26 & HAU, Hissar (216 m) & 49952 & 18 & 0.0 & $24.4-26.3 \times 22.5-24.4$ & $4 x$ & $\mathrm{ABN}$ \\
\hline P-27 & Gharaunda, Karnal (252 m) & 49953 & 18 & 69.2 & $22.5-24.4 \times 22.5-24.4$ & $4 x$ & $\mathrm{~N}$ \\
\hline P-28 & Narnaul, Mahendragarh (261 m) & 49954 & 18 & 55.2 & $24.4-26.3 \times 22.5-24.4$ & $4 x$ & $\mathrm{ABN}$ \\
\hline P-29 & Sanauli, Panipat $(218 \mathrm{~m})$ & 49955 & 18 & 70.2 & $24.4-26.3 \times 24.4-26.3$ & $4 x$ & $\mathrm{~N}$ \\
\hline P-30 & Devsar, Bhiwani (224 m) & 49956 & 18 & 45.0 & $24.4-26.3 \times 26.3$ & $4 x$ & $\mathrm{ABN}$ \\
\hline P-31 & Radaur, Yamunanagar $(255 \mathrm{~m})$ & 49957 & 18 & 39.2 & $24.4-28.1 \times 24.4$ & $4 x$ & $\mathrm{ABN}$ \\
\hline P-32 & Lalru, Ambala (272 m) & 49958 & 18 & 0.0 & $22.5-26.3 \times 22.5-24.4$ & $4 x$ & $\mathrm{ABN}$ \\
\hline P-33 & Naggal, Ambala (272 m) & 49959 & 18 & 46.7 & $22.5-24.4 \times 22.5-24.4$ & $4 x$ & $\mathrm{ABN}$ \\
\hline P-34 & Samalkha, Panipat (218 m) & 49960 & 18 & 32.6 & $22.5-26.3 \times 26.3$ & $4 x$ & $\mathrm{ABN}$ \\
\hline P-35 & Gohana, Sonipat (219m) & 49961 & 18 & 59.2 & $24.4-26.3 \times 26.3$ & $4 x$ & $\mathrm{~N}$ \\
\hline P-36 & Sunariya, Rohtak (213 m) & 49962 & 18 & 61.7 & $26.3-28.1 \times 22.5-26.3$ & $4 x$ & $\mathrm{~N}$ \\
\hline P-37 & Bahadurgarh, Jhajjar (219m) & 49963 & 18 & 32.4 & $26.3-28.1 \times 24.4-26.3$ & $4 x$ & $\mathrm{ABN}$ \\
\hline P-38 & Nizampur, Mahendragarh (261 m) & 49964 & 18 & 69.2 & $24.4-26.3 \times 26.3$ & $4 x$ & $\mathrm{~N}$ \\
\hline P-39 & Shahzadpur, Ambala (272 m) & 49965 & 18 & 58.4 & $24.4-26.3 \times 24.4-26.3$ & $4 x$ & $\mathrm{~N}$ \\
\hline P-40 & Parwanoo, Solan $(1350 \mathrm{~m})$ & 49966 & 18 & 29.2 & $22.5-24.4 \times 22.5-24.4$ & $4 x$ & $\mathrm{ABN}$ \\
\hline $\mathrm{P}-41$ & Trilokpur, Shiwaliks & 49967 & 18 & 64.9 & $22.5-24.4 \times 22.5-24.4$ & $4 x$ & $\mathrm{~N}$ \\
\hline P-42 & Kala amb, Ambala (272 m) & 49968 & 18 & 52.9 & $24.4-26.3 \times 24.4-26.3$ & $4 x$ & $\mathrm{~N}$ \\
\hline P-43 & Mandi Dabwali, Sirsa (204 m) & 49969 & 18 & 32.6 & $26.3-28.1 \times 26.3$ & $4 x$ & $\mathrm{ABN}$ \\
\hline P-44 & Gohana, Sonipat (219m) & 49970 & 18 & 19.4 & $24.4-26.3 \times 26.3$ & $4 x$ & $\mathrm{ABN}$ \\
\hline P-45 & Naror, Kaithal (219m) & 49971 & 18 & 15.7 & $24.4-28.1 \times 24.4-26.3$ & $4 x$ & $\mathrm{ABN}$ \\
\hline P-46 & Shahpur, Ambala (272 m) & 49972 & 18 & 62.8 & $24.4-26.3 \times 24.4-26.3$ & $4 x$ & $\mathrm{~N}$ \\
\hline P-47 & Nilokheri, Karnal (252 m) & 49973 & 18 & 25.6 & $22.5-24.4 \times 22.5-24.4$ & $4 x$ & $\mathrm{ABN}$ \\
\hline P-48 & Narwana, Jind (226 m) & 49974 & 18 & 74.9 & $26.3-28.1 \times 26.3-28.1$ & $4 x$ & $\mathrm{~N}$ \\
\hline P-49 & Jagadhri, Yamunanagar (255 m) & 49975 & 18 & 52.4 & $24.4-26.3 \times 24.4-26.3$ & $4 x$ & $\mathrm{~N}$ \\
\hline
\end{tabular}

$\mathrm{N}=$ Normal Meiotic course; $\mathrm{ABN}=$ Abnormal Meiotic course. 

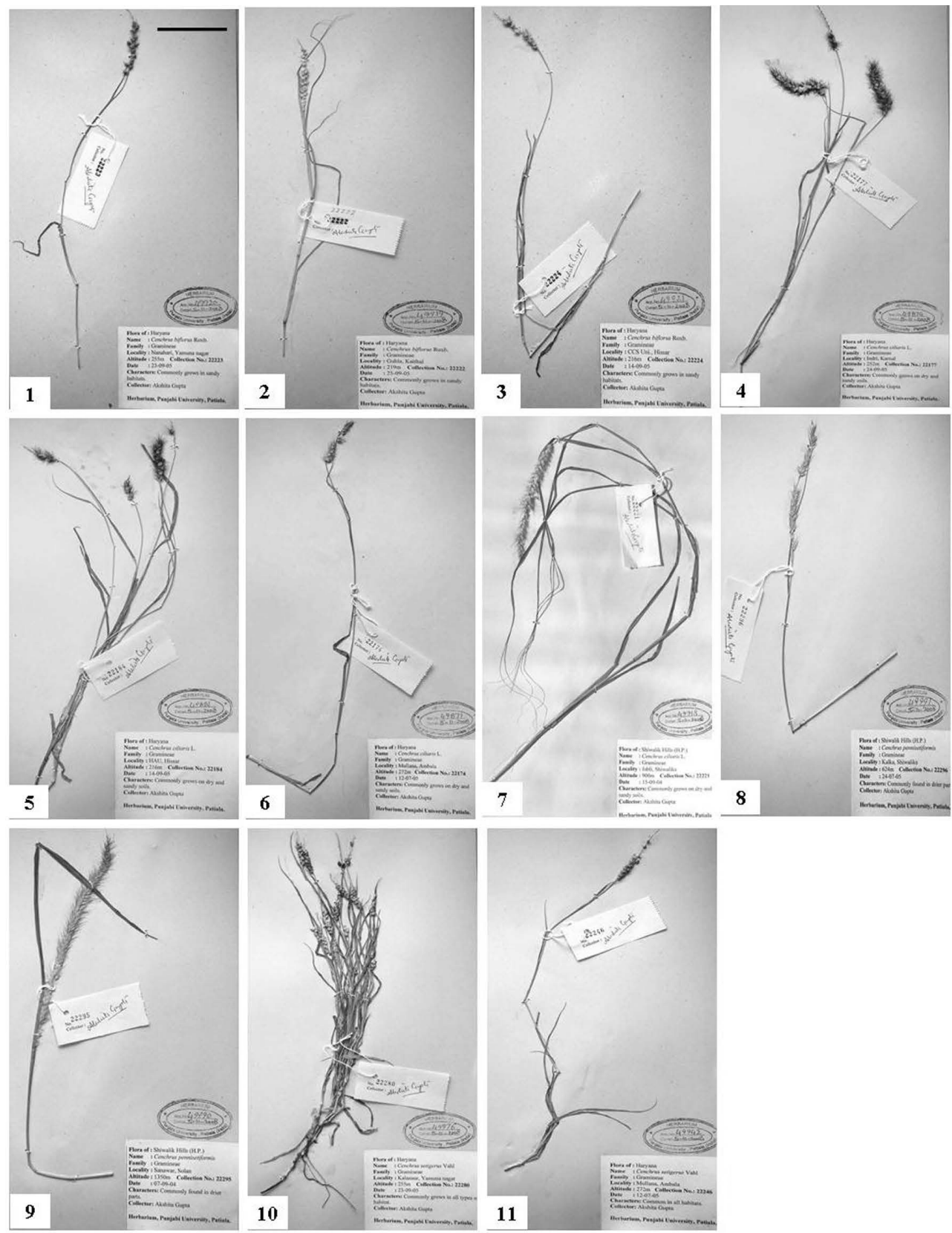

Figs. 1-11. Morphology of four species of Cenchrus on cytotype basis. Cenchrus biflorus, (1) Cytotype A ( $n=16)$, (2) Cytotype B $(n=17)$, (3) Cytotype C ( $n=18)$. C. ciliaris, (4) Cytotype A $(n=16)$, (5) Cytotype B $(n=17)$, (6) Cytotype C $(n=18)$, (7) Cytotype $\mathrm{D}(n=36)$. C. pennisetiformis, (8) Cytotype A $(n=17)$, (9) Cytotype $\mathrm{B}(n=18)$. C. setigerus, (10) Cytotype A $(n=17),(11)$ Cytotype B $(n=18)$. Scale bar $=10 \mathrm{~cm}$.

Results and discussion

During the present study four species i.e., C. biflorus, C. ciliaris, $C$. setigerus and $C$. pennisetiformis have been worked out cytologically. The meiosis of the pollen mother cells (PMCs) in the species is carried on population basis. The detailed meiosis with variation in morphological characters of each cytotype has been discussed in detail as under.

C. biflorus Roxb.: This species is common in sandy habitats and flowers during July-October. It is commonly known as Indian Sandbur, Bkut and Bhurat. Cytological studies have been made on 23 populations and three cytotypes with $n=16,17$ and $n=18$ are detected. The cytotype with $n=18$ is the most common. Morphologically, three cytotypes show fewer differences (Figs. 1-3, Table 2). Most of the populations of any cytotype show abnormal meiosis (Figs. 12-20). Meiotic abnormalities include, quadrivalents at diakinesis/metaphase-I (M-I), cytomixis and chromosome stickiness, laggards (zero to two per PMC) and bridges (zero to two per PMC). In some of the PMCs of the cytotype with $n=18$, unequal distribution of 19:17 is also present (Fig. 18). These meiotic abnormalities are reflected in the form of pollen sterility and variable pollen size (Table 2).

The earlier workers in India reported the normal mei- 

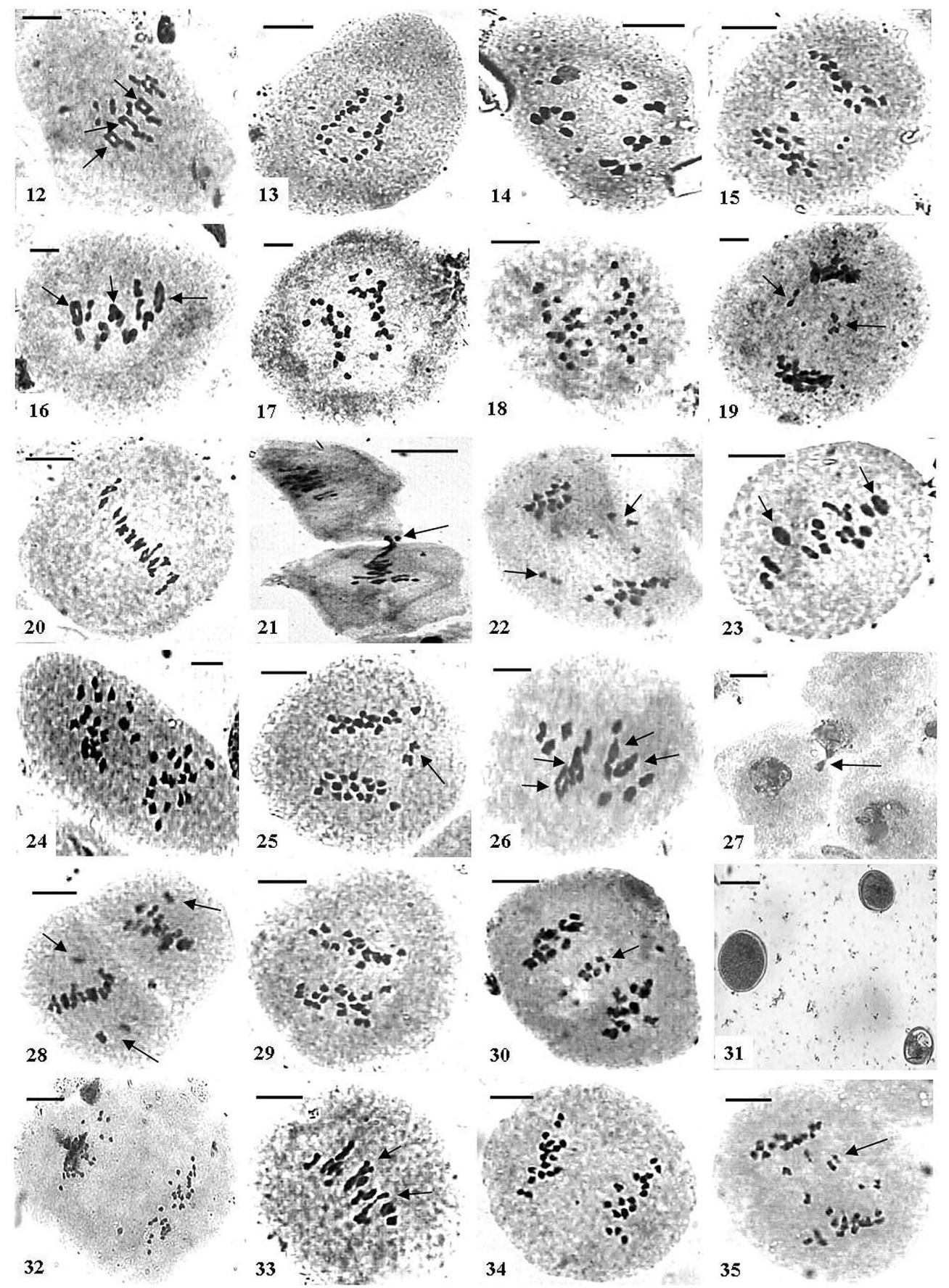

Figs. 12-35. Meiotic chromosome number, abnormalities and pollen grains. Cenchrus biflorus, (12) (Cytotype A) PMC at M-I showing $3_{\mathrm{IV}}+10_{\mathrm{II}}$ (arrow), (13) PMC at A-I with 16:16 chromosomes, (14) (Cytotype B) PMC at diakinesis showing 17 bivalents, (15) Laggards at A-I, (16) (Cytotype C) PMC at M-I with $3_{\mathrm{IV}}+12_{\mathrm{II}}$ (arrow), (17) PMC at A-I with 18:18 chromosomes, (18) PMC at A-I showing 19:17 chromosomes, (19) Laggards at A-I (arrow). C. ciliaris, (20) (Cytotype A) PMC at M-I with 16 ${ }_{\text {II }}$, (21) Cytomoxis (arrow), (22) Laggards at A-I (arrow), (23) (Cytotype B) PMC at M-I showing $2_{\mathrm{IV}}+13_{\mathrm{II}}$ (arrow), (24) PMC at A-I with 17:17 chromosomes, (25) Laggards at A-I (arrow), (26) (Cytotype C) PMC at M-I with $4_{\mathrm{IV}}+10_{\mathrm{II}}$ (arrow) (27) PMCs showing cytoplasmic connections (arrow), (28) Unoriented bivalents at M-I (arrow), (29) (Cytotype C) PMC showing 16:20 distribution, (30) Laggards at A-I (arrow), (31) Heterogenous sized pollen grains, (32) (Cytotype D) PMC at A-I showing 36:36 chromosomes, C. pennisetiformis, (33) (Cytotype A) PMC at M-I showing $2_{\mathrm{IV}}+13_{\mathrm{II}}$ (arrowed), (34) PMC at A-I showing 17:17 chromosomes, (35) Laggards (arrowed). Scale bar $=10 \mu \mathrm{m}$.

otic course in the species (Mehra 1982). The present reports of $n=16,17$ and 18 confirm the previous chromosome reports from India (Vij and Chaudhary 1981). The species exhibits intraspecific aneuploid cytotypes with $n=15,16,17,18$ and 24 .

C. ciliaris $\mathrm{L} .:$ It is also very common in sandy habitats, and flowers in July-October. Its common name is Bufflegrass. Forty eight populations have been studied 
Table 2. Morphological comparison of three cytotypes of C. biflorus.

\begin{tabular}{|c|c|c|c|}
\hline Characters & Cytotype A $(n=16)$ & Cytotype B $(n=17)$ & Cytotype C $(n=18)$ \\
\hline Distribution & Less frequent & Uncommon & Common \\
\hline Habit & Medium sized & Medium sized & Large \\
\hline Habitat & In neglected area and field & In neglected enclosures and fields & Common in waste places \\
\hline Plant height $(\mathrm{cm})$ & $34.0-35.5$ & $34.8-35.5$ & $47.3-66.0$ \\
\hline \multicolumn{4}{|l|}{ Leaf: } \\
\hline Size $(\mathrm{cm})$ & $7.2-7.5 \times 0.3-0.5$ & $19.5-20.0 \times 0.2-0.4$ & $10.9-26.0 \times 0.2-0.5$ \\
\hline Number & 2 & 4 & $4-5$ \\
\hline Sheath length (cm) & $5.5-6.0$ & $4.9-5.5$ & $7.1-12.5$ \\
\hline Number of nodes & 2 & 3 & $3-5$ \\
\hline \multicolumn{4}{|l|}{ Internode: } \\
\hline Length of uppermost internode $(\mathrm{cm})$ & $11.0-11.2$ & $8.5-8.8$ & $15.0-22.5$ \\
\hline Length of 2 nd internode from top $(\mathrm{cm})$ & $9.2-9.5$ & $5.2-5.6$ & $9.1-12.7$ \\
\hline \multicolumn{4}{|l|}{ Inflorescence: } \\
\hline Spike length (cm) & $6.9-7.1$ & $7.2-7.5$ & $5.7-12.9$ \\
\hline Length of spikelet (cm) & $5.5-5.6$ & $4.9-5.0$ & $7.1-7.5$ \\
\hline
\end{tabular}

Table 3. Morphological comparison of four cytotypes of C. ciliaris.

\begin{tabular}{|c|c|c|c|c|}
\hline Characters & Cytotype A $(n=16)$ & Cytotype B $(n=17)$ & Cytotype C $(n=18)$ & Cytotype $\mathrm{D}(n=36)$ \\
\hline Distribution & Less frequent & Uncommon & Frequently seen & Uncommon \\
\hline Habit & Medium sized & Medium sized & Medium sized & Tall \& tufted \\
\hline Habitat & $\begin{array}{l}\text { Neglected areas and } \\
\text { fields }\end{array}$ & $\begin{array}{c}\text { In neglected enclosures } \\
\text { and fields }\end{array}$ & Common in waste place & Dry sandy areas \\
\hline Plant height (cm) & $42.5-44.0$ & $48.0-49.5$ & $42.3-55.5$ & $80.0-95.0$ \\
\hline \multicolumn{5}{|l|}{ Leaf: } \\
\hline Size $(\mathrm{cm})$ & $11.2-11.5 \times 0.3-0.5$ & $16.8-17.2 \times 0.5-0.7$ & $9.1-15.0 \times 0.2-0.4$ & $49.8-50.0 \times 0.5-0.7$ \\
\hline Number & 4 & 5 & 3 & 5 \\
\hline Sheath length $(\mathrm{cm})$ & $6.2-6.5$ & $7.1-7.4$ & $6.2-6.5$ & $9.2-9.7$ \\
\hline Number of nodes & 3 & 4 & 3 & 5 \\
\hline \multicolumn{5}{|l|}{ Internode: } \\
\hline Length of upper most internode $(\mathrm{cm})$ & $10.5-10.7$ & $11.5-11.9$ & $12.2-12.5$ & $23.0-24.5$ \\
\hline Length of 2 nd internode from top $(\mathrm{cm})$ & $12.0-12.5$ & $9.5-9.7$ & $11.5-14.0$ & $10.2-11.0$ \\
\hline \multicolumn{5}{|l|}{ Inflorenscence: } \\
\hline Spike length (cm) & $5.8-6.0$ & $5.9-6.2$ & $4.6-5.3$ & $12.8-13.2$ \\
\hline Length of spikelet $(\mathrm{cm})$ & $1.5-1.7$ & $1.3-1.4$ & $1.1-1.4$ & $1.2-1.4$ \\
\hline
\end{tabular}

from different areas of Haryana and Shiwalik hills. Four cytotypes with $n=16,17,18$ and 36 are observed (Figs. $4-7)$. The tetraploid cytotype $(n=18)$, based on $x=9$, is better adapted to habitat and is the most common one as compared to the other cytotypes. The tetraploid and aneuploid cytotypes showed almost similar morphology, whereas the octaploid cytotype shows lot of gigantism (Table 3).

The meiotic course is found to be abnormal is all the three cytotypes $(n=16,17,18)$. The meiotic events in cytotypes with $n=16$ (Figs. 20-22) and $n=17$ (Figs. 23-25) show lot of abnormalities. In the cytotype $n=18,12$ populations show normal meiosis, whereas in 32 populations meiotic course is found to be abnormal due to the presence of quardrivalents at diakinesis/M-I (Fig. 26), cytomixis at prophase-I (Fig. 27) and M-I, chromosome stickiness and non-oriented bivalents at equatorial plate at M-I (Fig. 28), unequal distribution of chromosomes (Fig. 29) and laggards (Fig. 30), and bridges at A-I/T-I. The quadrivalents ranges from zero to four per PMC in different populations of all the cytotypes, and their average frequency per PMC also varies. The phenomena of cytomixis, non-oriented bivalents at equatorial plate and chromosome stickiness are present in few populations with varying frequency. The laggards (zero to four per PMC) and bridges (zero to two per PMC) are seen at A-I/T-I in fewer populations. Pollen fertility varies from almost sterile to $74.0 \%$ in the tetraploid cytotype and $54.7 \%$ in aneuploid cytotype (Table 1). However, octaploid cytotype (Fig. 31) shows normal meiosis with $82.7 \%$ pollen fertility. The pollen grains are of heterogenous size (Fig. 32) and show variation in different cytotypes.

All the present cytotypes are earlier known from India. Vij and Chaudhary (1981) reported the presence of B-chromosomes in the tetraploid cytotype $(2 n=36+0$ 2B) from Chandigarh. The species shows lot of variation in chromosome number showing both aneuploidy and polyploidy $(2 n=18,32,34,36,40,44,45,52,54,64,66$, 72 ) in the Indian populations. The normal seed setting in the population with pollen sterility might the apomictic nature of the species. The apomixis and polyembryony are well known in the species (Fisher et al. 1954). Present chromosome report of $2 n=36$ is in confirmation 

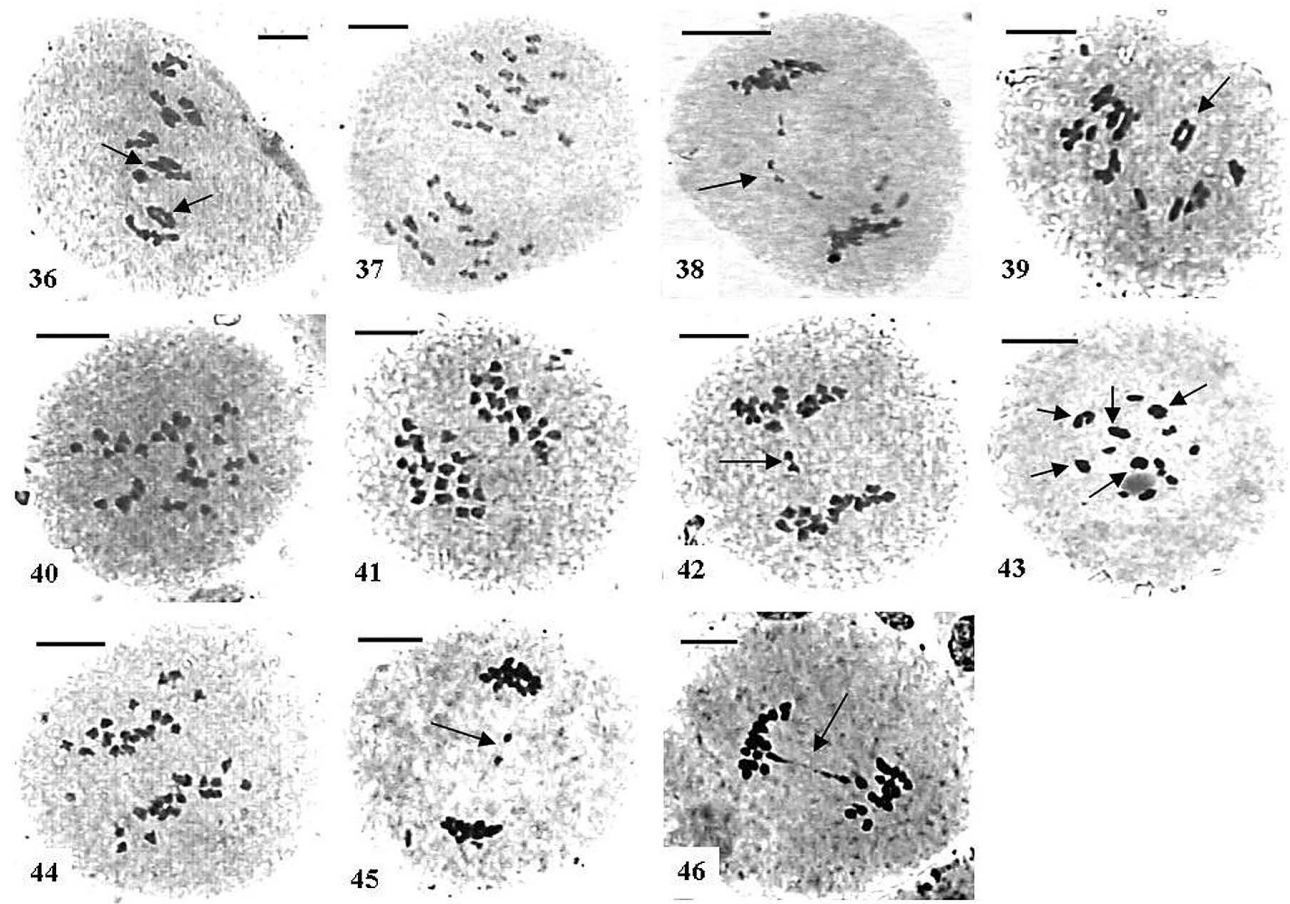

Figs. 36-46. C. pennisetiformis, (36) (Cytotype B) PMC at M-I showing $2_{\mathrm{IV}}+14_{\mathrm{II}}$ (arrow), (37) PMC at M-II with 18 chromosomes at each pole, (38) (Cytotype B) Laggards at A-I (arrow). C. setigerus, (39) PMC at M-I showing $1_{\mathrm{IV}}+15_{\mathrm{II}}$ (arrow), (40) PMC at A-I with 17:17 chromosomes, (41) PMC at A-I showing 16:18 chromosomes, (42) Laggards at A-I (arrow), (43) (Cytotype B) PMC at A-I with $5_{\mathrm{IV}}+8_{\mathrm{II}}$ (arrow), (44) PMC at A-I with 18:18 chromosomes, (45) Laggards at A-I (arrow), (46) Chromatin bridges at A-I (arrow). Scale $=10 \mu \mathrm{m}$.

Table 4. Morphological comparison of two cytotypes of $C$. pennisetiformis.

\begin{tabular}{|c|c|c|}
\hline Characters & Cytotype A $(n=17)$ & Cytotype B $(n=18)$ \\
\hline Distribution & Continued & Common \\
\hline Habit & Medium sized & Medium sized \\
\hline Habitat & $\begin{array}{c}\text { In neglected } \\
\text { enclosures and field }\end{array}$ & $\begin{array}{c}\text { Common in waste } \\
\text { places }\end{array}$ \\
\hline Plant height $(\mathrm{cm})$ & 37.2 & 36.8 \\
\hline \multicolumn{3}{|l|}{ Leaf: } \\
\hline Size $(\mathrm{cm})$ & 12.5 & 31.2 \\
\hline Sheath length (cm) & 3.2 & 5.3 \\
\hline Number of nodes & 2 & 1 \\
\hline Internodal length $(\mathrm{cm})$ & 9.1 & 10.2 \\
\hline \multicolumn{3}{|l|}{ Inflorescence: } \\
\hline Spike length (cm) & 4.4 & 4.3 \\
\hline Spikelet length $(\mathrm{cm})$ & 1.2 & 2.0 \\
\hline
\end{tabular}

with the earlier reports from India (Bir and Sahni 1985). The present count of $2 n=34$ is a new report for the species. The tetraploid cytotype $(2 n=36)$ is more common, whereas other cytotypes $(2 n=34,35,42,54)$ are relatively less common.

C. pennisetiformis Hochst. \& Steud.: It is commonly found in hotter and drier parts. The flowering period is July-October. It is a valuable fodder grass. Two cytotypes are reported with $n=17$ (Fig. 8) and $n=18$ (Fig. 9). The cytotypes are almost similar in morphological characters (Table 4). Meiosis is abnormal in both the cytotypes, with the presence of zero to three quadrivalents (Figs. 33, 36), besides normal bivalents at diakinesis/MI. At M-I, zero to four bivalents show non-orientation
Table 5. Morphological comparison of two cytotypes of C. setigerus.

\begin{tabular}{|c|c|c|}
\hline Characters & Cytotype A $(n=17)$ & Cytotype B $(n=18)$ \\
\hline Distribution & Less common & Common \\
\hline Habit & Medium sized & Medium sized \\
\hline Habitat & $\begin{array}{l}\text { In neglected area } \\
\text { and field }\end{array}$ & $\begin{array}{l}\text { Common in waste } \\
\text { places }\end{array}$ \\
\hline Plant height (cm) & $36.5-37.8$ & $37.6-38.2$ \\
\hline \multicolumn{3}{|l|}{ Leaf: } \\
\hline Size $(\mathrm{cm})$ & $6.3-6.7$ & $7.6-8.0$ \\
\hline Number & $4.9-5.2$ & $5.2-5.5$ \\
\hline Sheath length (cm) & 6 & 7 \\
\hline Number of nodes & 4 & 4 \\
\hline \multicolumn{3}{|l|}{ Internode: } \\
\hline $\begin{array}{l}\text { Length of uppermost } \\
\text { internode }(\mathrm{cm})\end{array}$ & $10.3-10.5$ & $9.8-10.2$ \\
\hline $\begin{array}{l}\text { Length of } 2 \text { nd internode } \\
\text { from top }(\mathrm{cm})\end{array}$ & $9.8-10.0$ & $8.2-8.5$ \\
\hline \multicolumn{3}{|l|}{ Inflorescence: } \\
\hline Spike length (cm) & $4.4-4.7$ & $4.3-4.6$ \\
\hline Length of spikelet (cm) & $0.4-0.7$ & $0.4-0.7$ \\
\hline
\end{tabular}

at equatorial plate in $30 \%$ of PMCs. The A-I mostly shows normal 17:17 distribution in cytotype with $n=17$ (Fig. 34) and zero to three laggards (Fig. 35) are seen in 23.6\% PMCs (P-1) and 19.7\% PMCs (P-2) at A-I/T-I. The pollen fertility is $27.8 \%$ in the tetraploid cytotype $\mathrm{B}$ $(n=18)$ and $19.9 \%$ in aneuploid cytotype with $n=17$. The pollen grain size also differs in the two cytotypes (Table $1)$.

C. setigerus Vahl.: This species is common in all type of habitats. It flowers in July-October. This species is 
a valuable pasture grass. Extensive cytological survey reveals the presence of $n=17$ and 18 (Figs. 10-11), show overlapping characters (Table 5). In the aneuploid cytotype with $n=17$, meiosis is also characterized by zero to one quadrivalent per PMC at diakinesis/M-I (Fig. 39), equal distribution of chromosomes i.e., 17:17 at A-I (Fig. 40), irregular distribution of chromosomes at A-I (16:18) (Fig. 41) and few laggards at A-I/T-I (Fig. $42)$ in all the presently studied populations. The cytotype with $n=18$ is normal in 14 populations, whereas 20 populations show abnormal meiosis due to the presence of zero to four quadrivalents per PMC at diakinesis/M-I (Fig. 43), 18:18 chromosomes at A-I (Fig. 44) and zero to three laggards per PMC at A-I/T-I (Fig. 45). In both the cytotypes, there is difference in pollen fertility and pollen grain size in different populations (Table 1). Patil et al. (1961) recorded $2 n=37$ from Rajasthan with the presence of $0-5_{\mathrm{IV}}, 0-1_{\mathrm{III}}, 8-18_{\mathrm{II}}$ and $0-3_{\mathrm{I}}$ in the species.

The present report of $2 n=34$ and 36 is in conformity with the various reports by different workers (Kaur and Gupta 2016a). Besides, these two cytotypes $(2 n=34$, 36), cytotypes with $2 n=37,54$ and 72 are known from India, and besides these, another cytotype $2 n=54$ is also known from outside India. Thus, the species shows lot of intraspecific aneuploidy and polyploidy.

From globe, the genus is cytologically known with 23 species and 60 cytotypes and six species with 30 cytotypes from Indian subcontinent. Chromosome number with in the genus varies from $2 n=18-102$, of which $2 n=34$ is the dominating cytotype $(32.78 \%)$, followed by $2 n=36(11.47 \%)$. Earlier, the genus was said to be monobasic with $x=9$ as the primary base number (Brown 1948), further dibasic with $x=9$ and $x=17$ (Darlington and Wylie 1955). Now a days, genus is polybasic including $x=7,9,12$ and 17 as basic chromosome numbers (De Lisle 1964, Kharrat-Suissi et al. 2013). Vij and Chaudhary (1981) suggested that $2 n=34$ was derived by a loss of pair of chromosomes from $2 n=36$. During the present study, irregular meiosis has been observed with differed frequency of various chromosomal anomalies. The presence of multivalents along with bivalents at diakinesis and M-I in all the accessions of the presently studied species have been observed. Similar results were given by Nath et al. (1970) in C. ciliaris and some other members of the tribe Paniceae, and also suggested that they were autopolyploid in origin.

\section{Acknowledgements}

The authors are thankful to DBT-IPLS project
(Project no. BT/PR-4548/INF/22/146/2012) sanctioned to Punjabi University, Patiala and UGC BSR Indira Gandhi Single Girl Child fellowship to Navjot Kaur (Award letter number: F.7-152/2007 BSR) for financial assistance. The authors are also thankful to the Head of Department of Botany, Punjabi University, Patiala for providing necessary laboratory facilities.

\section{References}

Bhat, V., Dalton, S. J., Kumar, S., Bhat, B. V., Gupta, M. G. and Morris, P. 2001. Particle-inflow-gun-mediated genetic transformation of buffel grass (Cenchrus ciliaris L.): optimizing biological and physical parameters. J. Appl. Genet. 42: 405-412.

Bir, S. S. and Sahni, M. 1985. Cytological investigations on some grasses from Punjab Plain, North India. Proc. Indian Sci. Natl. Acad. B51: 609-626.

Bor, N. L. 1960. The Grasses of Burma, Ceylon, India and Pakistan. Pergamon Press, New York.

Brown, W. V. 1948. A cytological study in Gramineae. Am. J. Bot. 35: 382-395.

Clayton, S. D. and Renvoize, S. A. 1986. Genera Graminum. Grasses of the World. Royal Botanic Gardens, Kew, London.

Darlington, C. D. and Wylie, A. P. 1955. Chromosome Atlas of Flowering Plants. Allen and Unwin Ltd., London.

De Lisle, D. G. 1964. Chromosome numbers in Cenchrus (Gramineae). Am. J. Bot. 51: 1133-1134.

Fisher, W. D., Bashaw, E. C. and Holt, E. C. 1954. Evidence for apomixes in Pennisetum ciliare and Cenchrus setigerus. Agron. J. 46: 401-404.

Gupta, R. C., Gupta, A. and Kaur, N. 2017. Meiotic studies in some members of tribe Andropogoneae (Poaceae) from semi desert area of North India. Cytologia 82: 105-113.

Kaur, N. and Gupta, R. C. 2016a. Cytological study in some members of tribe Paniceae (Poaceae) from Rajasthan. Cytologia 81: 13-17.

Kaur, N. and Gupta, R. C. 2016b. Anomalous meiotic behavior in different populations of octaploid Sorghum bicolor (L.) Moench from Sirohi District (Rajasthan), India. Chromosome Bot. 11: 21-26.

Kharrat-Souissi, A., Siljak-Yakovlev, S., Brown, S. C. and Chaieb, M. 2013. Cytogeography of Cenchrus ciliaris (Poaceae) in Tunisia. Folia Geobot. 48: 95-113.

Mehra, P. N. 1982. Cytology of East Indian Grasses. Pramodh P. Kapur at Rajbandhu Industrial Company, New Delhi.

Moulik, S. 1997. The Grasses and Bamboos of India. Volumes 1 and 2. Scientific Publishers, Jodhpur.

Nath, J., Swaminathan, M. S. and Mehra, K. L. 1970. Cytological studies in the tribe Paniceae, Gramineae. Cytologia 35: 111-131.

Patil, B. D., Vohra, S. K. and Joshi, A. B. 1961. Chromosome numbers in some forage grasses. Curr. Sci. 30: 393-394.

Tzvelev, N. N. 1989. The system of grasses (Poaceae) and their evolution. Bot. Rev. 55: 141-204.

Vij, S. P. and Chaudhary, J. D. 1981. Cytological investigations in three species of Cenchrus L. (Gramineae). Cytologia 46: 661-669.

Watson, L. and Dallwitz, M. J. 1992. The Grass Genera of the World. CAB International, Wallingford. 